\title{
具有高价氧化态的超薄二维Ni-MOF高性能电催化 剂用于苯甲醇氧化反应
}

\author{
袁萌伟 ${ }^{1,3}$, 李雅银 ${ }^{1}$, 孙泽民 ${ }^{2}$, 孙根班 ${ }^{1,2 *}$ \\ 1. 北京师范大学化学学院, 能量转换与存储材料北京市重点实验室, 北京 100875 \\ 2. 北京师范大学珠海校区自然科学高等研究院, 先进材料研究中心, 珠海 519087 \\ 3. 北京师范大学物理学系, 应用光学北京市重点实验室, 北京 100875 \\ *通讯作者, E-mail: gbsun@bnu.edu.cn
}

收稿日期: 2021-12-29; 接受日期: 2022-02-09; 网络版发表日期: 2022-02-17

国家自然科学基金(编号: 21771024)、中国博士后科学基金(编号: M2020680430)资助项目

摘要超薄二维金属有机框架(Two-dimensional metal-organic frameworks (2D MOFs))具有较大的比表面积和 开放的催化活性中心，在电催化反应中具有潜在的应用价值. 本工作报道了一种溶剂热法制备的具有开放位点的 超薄2D Ni-MOF纳米片阵列及其在电催化苯甲醇氧化(Electrocatalytic benzyl-alcohol oxidation (EBO)) 方面的应 用. 作为一种高性能的EBO电催化剂, $2 \mathrm{D} \mathrm{Ni}-\mathrm{MOF}$ 表现出较低的过电位和优异的化学耐久性, 仅需要约 $1.50 \mathrm{~V}$ 的 电位即可达到 $250 \mathrm{~mA} \mathrm{~cm}^{-2}$, 苯甲醇氧化效率达 $85 \%$. 2D Ni-MOF纳米片阵列相比于同样具有超薄结构的 $2 \mathrm{D} \mathrm{Ni}$ $(\mathrm{OH})_{2}$ 纳米片阵列, 反应过电势降低约 $35 \mathrm{mV}\left(@ 50 \mathrm{~mA} \mathrm{~cm}^{-2}\right)$, 具有更快的反应动力学. 在同一恒电位下进行苯甲 醇氧化反应时, 具有更高的响应电流, 并且在连续电解 $20000 \mathrm{~s}$ 后, 可保留 $25 \mathrm{~mA} \mathrm{~cm}^{-2}$ 的电流密度, 接近 $\mathrm{Ni}(\mathrm{OH})_{2}$ 纳 米片的两倍, 表现出更优异的电催化活性. Ni-MOF纳米片中的 $\mathrm{Ni}$ 具有更高的氧化态, 在EBO反应中更容易转化成 活性位点 $\mathrm{NiOOH}$, 使得其在更低的电位下能有效催化苯甲醇的电氧化反应. 这一结果为进一步设计高性能电催化 苯甲醇氧化反应电催化剂提供新的视角.

关键词超薄二维结构, 二维金属有机框架, 苯甲醇氧化, 电催化剂, 高价氧化态

\section{1 引言}

随着能源和环境问题的日益突出，开发利用可再 生清洁能源十分必要. 氢能是一种极具发展前景的可 再生清洁能源, 电解水是获得氢气的重要途径 ${ }^{[1 \sim 6]}$. 电 解水的理论电压为 $1.23 \mathrm{~V}$, 实验中, 即使在添加高性能 的电催化剂时, 依然需要 $1.50 \mathrm{~V}$ 左右的电压才能实现
水的全解(@10 $\left.\mathrm{mA} \mathrm{cm})^{-2}\right)^{[7]}$. 电解水产氢的阳极产物 为氧气, 本身的附加值并不高. 因此, 电解混合水产氢, 例如尿素和苯甲醇等, 同时生产高附加值化学品是一项 节约资源、保护环境, 且富有实用价值的有效策略 ${ }^{[8 \sim 12]}$.

目前，工业上生产苯甲酸 $(\mathrm{Ph}-\mathrm{COOH})$ 几乎完全依 赖于能源和资源密集型甲苯氧化工艺，采用高压 $(1 \mathrm{MPa}) 、$ 高温 $\left(150 \sim 170^{\circ} \mathrm{C}\right)$ 和有毒化学氧化剂来克服

引用格式: Yuan M, Li Y, Sun Z, Sun G. Ultrathin two-dimensional Ni-MOF with high-valence oxidation states as high-performance electrocatalytic catalysts for benzyl-alcohol oxidation. Sci Sin Chim, 2022, 52: 913-922, doi: 10.1360/SSC-2021-0262 
动力学的限制，但会造成严重的环境污染和资源浪 费 ${ }^{[8]}$. 开发环境友好且持续的新型 $\mathrm{Ph}-\mathrm{COOH}$ 生产方法 对于工业的绿色发展、缓解日益突出的资源问题和日 益严重的环境问题具有重要意义. 因此, 电催化氧化苯 甲醇与产氢相结合的多功能混合水电解工艺，由于其 操作条件温和、热力学势垒低、工艺节能，可以实现 高附加值化学品(氢气和苯甲酸)的同时生产，以及能 源的进一步有效利用. 在反应热力学方面, EBO比析 氧反应(oxygen evolution reaction, OER)更有优势，具 有更低的理论电势. 然而, 如果要达到工业生产所需的 电流密度，例如超过 $350 \mathrm{~mA} \mathrm{~cm}{ }^{-2}$, OER和EBO之间的 竞争将成为不可避免的问题，这将大大降低EBO反应 的效率. 为了实现能够在大电流密度下运行的混合水 电解，电催化剂需要具有高的EBO性能. 近年来，Yin 等人 ${ }^{[13]}$ 研究发现相比于 $\mathrm{Co}_{3} \mathrm{O}_{4}$ 纳米颗粒, $\mathrm{Co}_{3} \mathrm{O}_{4}$ 纳米针 阵列具有出更好的EBO活性. 此外, Huang等人 ${ }^{[14]}$ 通过 界面工程在 $\mathrm{Fe} / \mathrm{Co}$ 氧化物中构建异质结构, 引发局部结 晶和缺陷氧的产生, 并且在 $10 \mathrm{~mA} \mathrm{~cm}^{-2}$ 下具 $1.42 \mathrm{~V}$ 的 较低电位. 因此, 对材料的形貌、组成、结构等进行 目标性的调控，有望实现EBO性能的有效提升，有助 于电催化产氢时高附加值化学品的获取.

金属有机框架材料是有机配体和金属离子组成的 二级结构单元, 由于其中心离子和配体种类多样, 因此 本身结构具有高度可调性 ${ }^{[15,16]}$ 。通过对金属离子和配 体的调控，可以赋予MOFs不同的性能，因而被广泛的 应用于催化、分离和吸附等领域 ${ }^{[1721]}$. 由于其特殊的 结构特点, MOFs 具有作为均相和非均相催化剂的潜 能. 同时, MOFs固有的传输路径和均匀的多孔结构, 使其具有较高的气体可接近性、扩散性和催化活 性 ${ }^{[22,23]}$. 作为典型的低维MOFs之一, 2D MOFs 在电催 化领域具有广阔的发展前景，其超薄的二维结构不仅 继承了MOFs的特性，而且在表面上还具有高比例的 暴露金属原子 ${ }^{[21,24]}$. 这些金属原子具有许多悬空键和 配位不饱和金属位, 这对电催化具有重要作用. 此外, 纳米厚度的超薄2D MOFs与传统二维材料类似，具有 较大的比表面积和丰富的活性中心，以促进快速电子 转移, 确保催化剂和电解质之间的紧密接触以及快速 传质 ${ }^{[19,25 ~ 29]}$. 因此，2D MOFs 可作为一种理想的电催 化剂, 在电催化中已经展现出良好的活性和应用, 例如 $\mathrm{OER}^{[1]}$ 、锂空气电池等 ${ }^{[18]}$ ，但超薄2D MOFs 在EBO中 的应用尚属空白.
本工作首次将超薄 $2 \mathrm{D} \mathrm{Ni-MOF}$ 作为电催化剂用 于电催化苯甲醇氧化反应，仅需要约 $1.50 \mathrm{~V}$ 的电位即可 达到 $250 \mathrm{~mA} \mathrm{~cm}^{-2}$, 比2 $\mathrm{D} \mathrm{Ni}(\mathrm{OH})_{2}$ 纳米片高 $100 \mathrm{~mA} \mathrm{~cm}^{-1}$. 且反应过电势比其低约 $35 \mathrm{mV}\left(@ 50 \mathrm{~mA} \mathrm{~cm}^{-2}\right)$, 具有更 优异的电催化活性. 同时, Ni-MOF具有更高的响应电 流, 连续电解 $20000 \mathrm{~s}$ 后, 可保留 $25 \mathrm{~mA} \mathrm{~cm}$ 的电流密 度, 接近 $\mathrm{Ni}(\mathrm{OH})_{2}$ 纳米片的两倍. 以 $\mathrm{Ni}-\mathrm{MOF}$ 纳米片为 催化剂, 在经过长时间的电解后, 可得到 2 倍于 $\mathrm{Ni}(\mathrm{OH})_{2}$ 纳米片催化剂的苯甲酸产物. Ni-MOF纳米片 中 $\mathrm{Ni}$ 具有更高的氧化态，在 $\mathrm{EBO} 反$ 应中更容易转化成

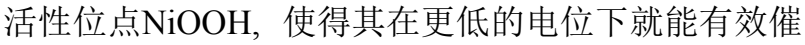
化苯甲醇的电催化反应.

\section{2 实验部分}

\section{1 实验药品}

$\mathrm{NiCl}_{2} \cdot 6 \mathrm{H}_{2} \mathrm{O}$ (分析纯, 天津市博迪化工股份有限公 司), $\mathrm{Ni}\left(\mathrm{NO}_{3}\right)_{2} \cdot 6 \mathrm{H}_{2} \mathrm{O}$ (分析纯, 天津市科密欧化学试剂 有限公司), $\mathrm{KOH}$ (分析纯, 国药集团化学试剂有限公 司), $N, N$-二甲基甲酰胺 (分析纯, 天津富宇精细化工有 限公司), 乙醇 (分析纯，北京市通广精细化工公司), $\mathrm{NH}_{4} \mathrm{~F}$ (分析纯，北京市通广精细化工公司), $\mathrm{N}_{2} \mathrm{H}_{4} \mathrm{CO}$ (分析纯, 北京市通广精细化工公司), 浓盐酸 (分析纯, 北京市通广精细化工公司), 泡沫镍 (深圳天成和科技 有限公司).

\section{2 电催化剂合成}

首先, 在 $3 \mathrm{~mol} \mathrm{~L}^{-1}$ 盐酸溶液中, 超声波清洗泡沫镍 $\left(4 \times 6 \mathrm{~cm}^{2}\right)$. 然后, 在丙酮和去离子水 $(1: 2)$ 的混合物中超 声10分钟, 并用去离子水冲洗三次备用.

溶剂热法制备 $2 \mathrm{D} \mathrm{Ni}(\mathrm{OH})_{2}$ 纳米片阵列. 首先, 将 $3.2 \mathrm{mmol} \mathrm{Ni}\left(\mathrm{NO}_{3}\right)_{2} \cdot 6 \mathrm{H}_{2} \mathrm{O} 、 3.2 \mathrm{mmol}$ 氟化铵和 $8 \mathrm{mmol}$ 尿素加入 $80 \mathrm{~mL}$ 去离子水中并搅拌至溶解, 放入泡沫 镍基底. 然后，将装有上述混合溶液的高压釜放入烘 箱中加热反应 $\left(120^{\circ} \mathrm{C}, 5 \mathrm{~h}\right)$. 最后，将合成好的样品用 乙醇和去离子水洗涤, 并在真空烘箱中干燥.

溶剂热法制备2D Ni-MOF纳米片阵列. 将 $32 \mathrm{~mL}$ $N, N$-二甲基甲酰胺、 $2 \mathrm{~mL}$ 乙醇和 $2 \mathrm{~mL}$ 去离子水混合 形成均相溶液. 然后, 向上述溶液中加入 $4 \times 6 \mathrm{~cm}^{2}$ 泡沫 镍、 $0.75 \mathrm{mmol}$ 对苯二甲酸和 $0.75 \mathrm{mmol} \mathrm{NiCl}_{2} \cdot 6 \mathrm{H}_{2} \mathrm{O}$, 摚拌至溶解. 然后将混合物放入高压釜中 $140^{\circ} \mathrm{C}$ 反应 
$12 \mathrm{~h}$. 最后，将所制备好的样品，用乙醇和去离子水洗 涤数次, 然后真空干燥, 留存备用.

\section{3 催化剂表征}

通过场发射扫描电子显微镜(Field-emission scanning electron microscope, SEM, SU-8010, 日立)对所制 备材料的结构和形貌进行了表征。利用透射电镜 (Transmission electron microscope, TEM, 80/200 kV加 速电压, JEM-2010, JEOL)表征所合成催化剂的微观结 构. 通过X射线光电子能谱(X-ray photoelectron spectroscopy, XPS, ESCALAB 250Xi)和X射线衍射(X-ray diffraction，XRD)进行表面组成分析和晶体结构表征. 利用核磁共振仪 ${ }^{1} \mathrm{H}$ nuclear magnetic resonance (NMR), Bruker, $500 \mathrm{MHz}$ )对电解后的阳极产物进行结构表征.

\section{4 电化学性能测试}

在标准的三电极系统中 $(\mathrm{Pt}$ 片作为对电极, $\mathrm{Hg}$ / $\mathrm{HgO}$ 电极作为参比电极, 所合成的催化剂作为工作电 极), 使用电化学工作站(CHI601E)进行电化学性质测 量. 利用线性扫描伏安(LSV)曲线测试了在 $1.0 \mathrm{M}$ $\mathrm{KOH}$ 中 OER的性能和在 $1.0 \mathrm{M} \mathrm{KOH}+0.1 \mathrm{M}$ 苯甲醇
(BA)中的EBO反应性能，扫描速率为 $10 \mathrm{mV} \mathrm{s}^{-1}$. 根据 以下公式将电势转换为可逆氢电极 $(\mathrm{RHE}): E_{\mathrm{RHE}}=$ $E_{\mathrm{Hg} / \mathrm{HgO}}+0.098+0.0591 \times \mathrm{pH}$. 在 $1.42 \mathrm{~V}$ 下测试计时电流 曲线 $(i-t)$, 以考察催化剂的稳定性. 双电层电容值 $\left(C_{\mathrm{dl}}\right)$ 采用不同扫速下的循环伏安曲线测试得到，测试范 围为 $1.027 \sim 1.077 \mathrm{~V}$ ，扫速分别为 $10 、 20 、 30 、 40$ 和 $50 \mathrm{mV} \mathrm{s}^{-1}$.

\section{5 电催化产物分析表征}

在1.42 V下, $1.0 \mathrm{M} \mathrm{KOH}+0.1 \mathrm{M} \mathrm{BA}$ 溶液中 $(80 \mathrm{~mL})$, 连续电解 $30 \mathrm{~h}$. 将电解后的溶液用浓盐酸酸化, 静置 $24 \mathrm{~h}$ 后, 对所得晶体进行过滤、收集, $70^{\circ} \mathrm{C}$ 下, 真空干 燥 $12 \mathrm{~h}$.

\section{3 结果与讨论}

\section{1 催化剂表征}

图 $1 \mathrm{a}$ 和 $\mathrm{b}$ 是所合成的 $2 \mathrm{D} \mathrm{Ni}-\mathrm{MOF}$ 在不同放大倍数 下的SEM照片，从图中可以看出 $\mathrm{Ni}-\mathrm{MOF}$ 的横向尺寸 约2 5 $\mu \mathrm{m}$ ，厚度小于 $10 \mathrm{~nm}$ ，呈典型的纳米片状形貌， 且均匀地生长在泡沫镍基底上，形成Ni-MOF纳米片
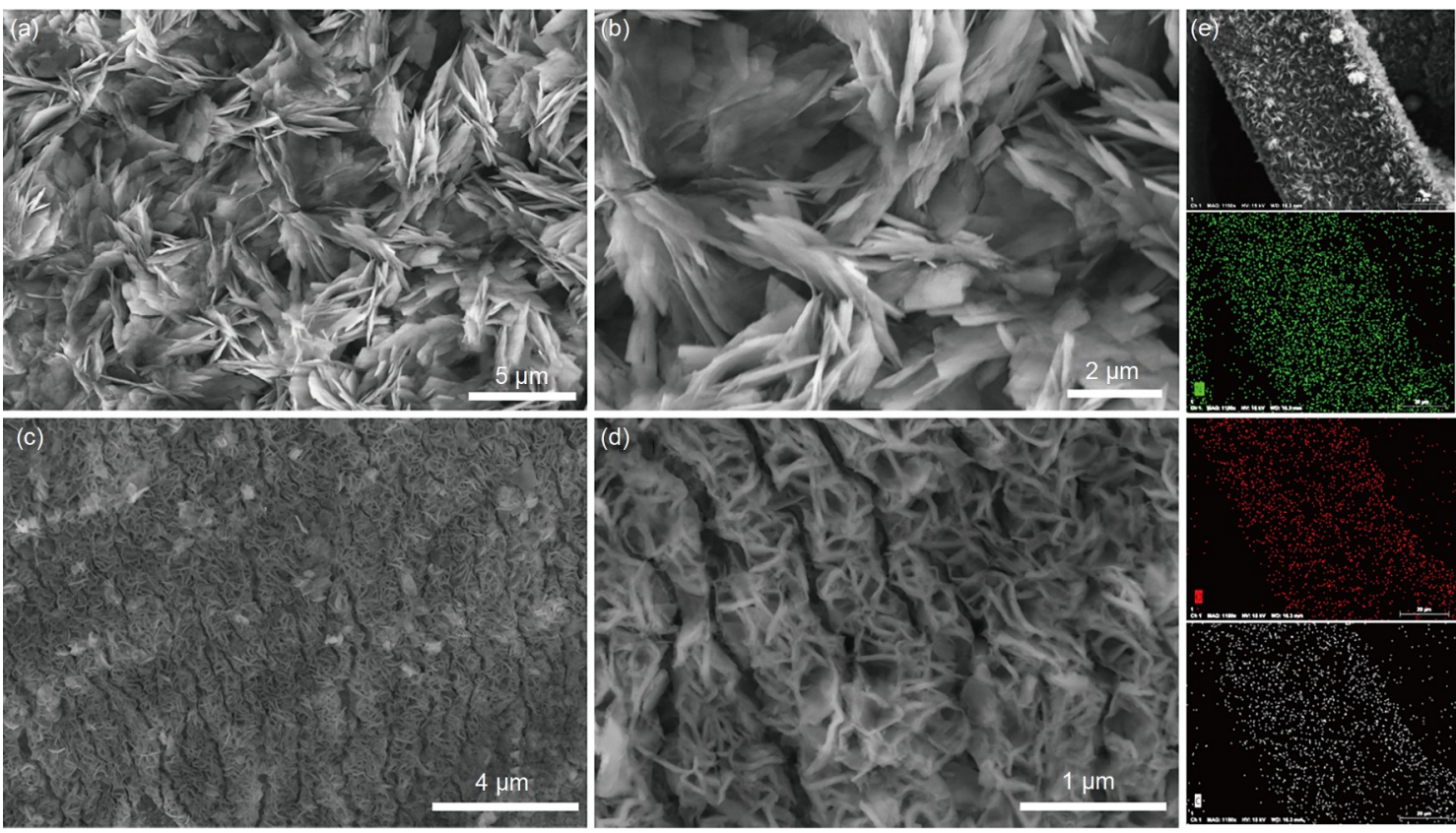

图 1 超薄2D (a, b) Ni-MOF纳米结构和 $(\mathrm{c}, \mathrm{d}) \mathrm{Ni}(\mathrm{OH})_{2}$ 纳米结构的SEM照片; (e) 超薄2D Ni-MOF纳米结构的元素mapping图 (网络版彩图)

Figure 1 The SEM images of the ultrathin 2D (a, b) Ni-MOF and (c, d) $\mathrm{Ni}(\mathrm{OH})_{2}$ electrocatalysts. (e) The elemental mapping of the ultrathin 2D NiMOF nanostructure (color online). 
阵列结构. 图 $1 \mathrm{c}$ 和 $\mathrm{d}$ 为 $2 \mathrm{D} \mathrm{Ni}(\mathrm{OH})_{2}$ 的 $\mathrm{SEM}$ 照片, 图中清 楚地显示 $2 \mathrm{D} \mathrm{Ni}(\mathrm{OH})_{2}$ 为堆积紧密的纳米片阵列结构. 与 $2 \mathrm{D} \mathrm{Ni-MOF}$ 结构相似, 纳米片间均呈现出明显的孔 洞, 可充分暴露活性位点, 有利于电解液的润湿和反应 物分子的扩散, 可促进电催化反应的进行. 图 $1 \mathrm{e}$ 为 $\mathrm{Ni}-$ MOF纳米片的相应元素mapping图，其中Ni-MOF由 C、Ni、O元素组成, 且这些元素均匀分布在整个纳米 片阵列中, 可证明 Ni-MOF的成功制备.

图2a为2D Ni-MOF的TEM照片, Ni-MOF呈现明 显的超薄纳米片状结构, 与SEM照片结果一致. 图 $2 \mathrm{~b}$ 为 所合成2D Ni-MOF的HRTEM照片，图中呈现明显的 单向晶格条纹, 晶格条纹间距为 $1.06 \mathrm{~nm}$ (晶格间距值 为 10 个间距的平均值), 对应 $2 \mathrm{D} \mathrm{Ni-MOF}$ 的(200)晶面. 图 $2 \mathrm{c}$ 和 $\mathrm{d}$ 为 $2 \mathrm{D} \mathrm{Ni}(\mathrm{OH})_{2}$ 的不同放大倍数下的透射电镜 照片. 图 $\mathrm{c}$ 中的 $\mathrm{Ni}(\mathrm{OH})_{2}$ 纳米片显示出明显的堆叠, 主 要原因可能是纳米片阵列本身生长时比较致密, 导致 相互交联，从而呈现出多级交错的结构 (图2d). 图 $2 \mathrm{e}$ 为 $2 \mathrm{D} \mathrm{Ni}(\mathrm{OH})_{2}$ 的高分辨透射电镜照片, 显示出清晰的
晶格条纹，条纹间距为 $0.254 \mathrm{~nm}$, 对应 $\mathrm{Ni}(\mathrm{OH})_{2}$ 的 (111) 晶面(JCPDS No. 14-0117).

图3a和 $\mathrm{b}$ 为 $\mathrm{Ni}-\mathrm{MOF}$ 和 $\mathrm{Ni}(\mathrm{OH})_{2}$ 纳米结构的 XRD图 谱, Ni-MOF的衍射峰结果与文献报道结果一致, 且无 额外衍射峰的出现, 证明所制备 $\mathrm{Ni}-\mathrm{MOF}$ 基本为纯 相 $^{[16]}$. 在 $\mathrm{Ni}(\mathrm{OH})_{2}$ 的XRD谱图中, 两种晶相共存(JCPDS No. 14-0117和JCPDS No. 22-0444). 利用XPS进一步分 析了氢氧化镍和Ni-MOF纳米结构的表面组成和元素 价态, 结果如图 $3 \mathrm{c} \sim \mathrm{i}$ 所示, 图 $3 \mathrm{c}$ 和 $d$ 为 $\mathrm{Ni}-\mathrm{MOF}$ 和 $\mathrm{Ni}(\mathrm{OH})_{2}$ 纳米结构的XPS全谱图, 图3e i 为相应元素的 精细谱(精细谱是以C $1 \mathrm{~s} 284.8 \mathrm{eV}$ 为基准, 能量分度值 为 $0.05 \mathrm{eV}$, 对不同高分辨谱进行电荷矫正得到的). 图 $3 \mathrm{e}$ 为 $\mathrm{Ni}-\mathrm{MOF}$ 的 C $1 \mathrm{~s}$ 谱, 结合能在 284.8、286.1和 $288.4 \mathrm{eV}$ 处的峰分别归属于 $\mathrm{C}-\mathrm{C} / \mathrm{C}=\mathrm{C}$ 键、 $\mathrm{C}-\mathrm{O}$ 键和 $\mathrm{C}=\mathrm{O}$ 键，与 $\mathrm{Ni}-\mathrm{MOF}$ 中配体中对苯二甲酸的官能团相 对应 ${ }^{[30]}$. 图3f中, Ni $2 p_{3 / 2}$ 和 $\mathrm{Ni} 2 \mathrm{p}_{1 / 2}$ 的结合能为 856.2 和 $873.9 \mathrm{eV}$, 与文献报道结果一致, 表明 $\mathrm{Ni}^{2+}$ 在 $\mathrm{Ni}-\mathrm{O}$ 键中 的存在 ${ }^{[18]}$. 其中, $\mathrm{Ni} 2 \mathrm{p}_{3 / 2}$ 表现出比 $\mathrm{Ni}(\mathrm{OH})_{2}$ 中 $\mathrm{Ni} 2 \mathrm{p}_{3 / 2}$ 更
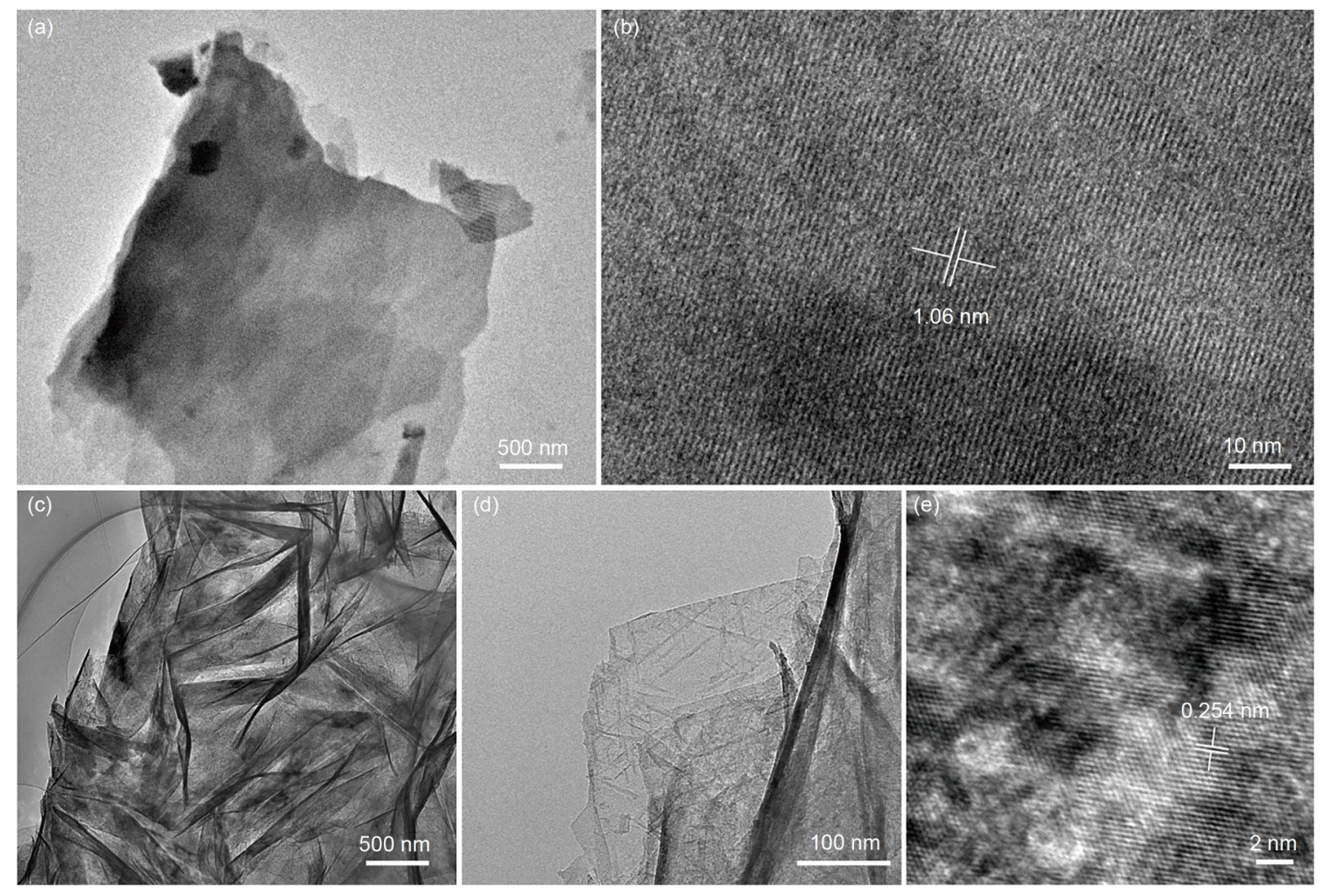

图 2 超薄2D Ni-MOF纳米结构的(a) TEM照片和(b) 高分辨透射电镜(HRTEM)照片; 超薄2D Ni(OH) $)_{2}$ 纳米结构的(c, d) TEM 照片和(e) HRTEM照片 (网络版彩图)

Figure 2 The (a) TEM and (b) HRTEM images of the ultrathin 2D Ni-MOF. The (c, d) TEM and (e) HRTEM images of the ultrathin 2D Ni(OH) 2 (color online). 

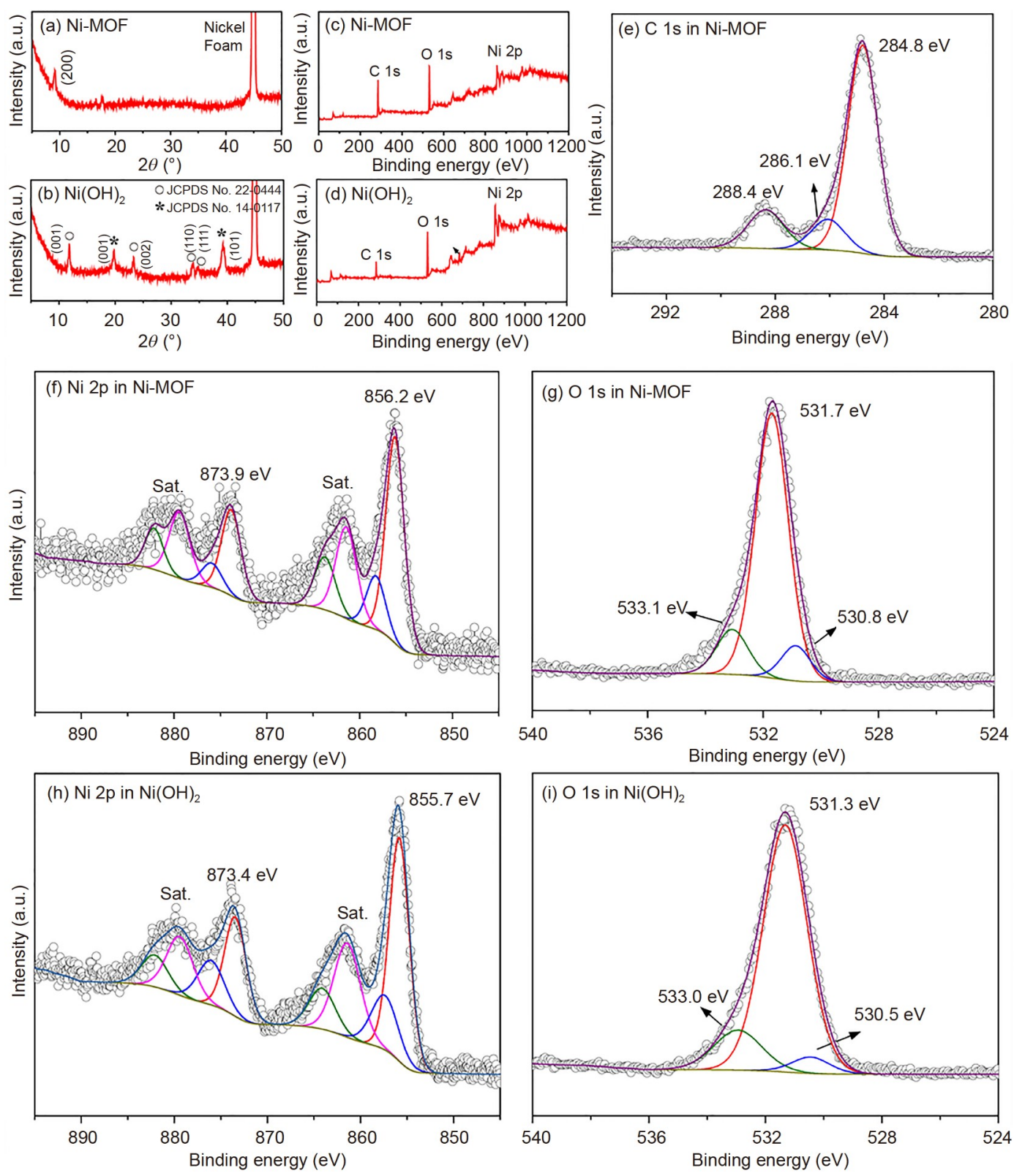

图 3 超薄2D Ni-MOF和 $\mathrm{Ni}(\mathrm{OH})_{2}$ 纳米结构的XRD图谱 $(\mathrm{a}, \mathrm{b}), \mathrm{XPS}$ 全谱 (c, d) 和C 1s, Ni 2p, O 1s的高分辨谱(e i) (网络版彩图) Figure 3 The XRD patterns (a, b), XPS survey spectra (c, d) and high-resolution C 1s, Ni 2p, O 1s spectra (e-i) of the ultrathin 2D Ni-MOF and $\mathrm{Ni}(\mathrm{OH})_{2}$ (color online).

高的氧化态 (图3h, $855.7 \mathrm{eV}$ ), 在电催化氧化反应中, 将 更利于向高价态的活性物质转化，表现出更好的催化 活性. 图3g和i的O $1 \mathrm{~s}$ 谱中, $531.7 \mathrm{eV}$ 和 $531.3 \mathrm{eV}$ 处的结 合能对应不同样品中的金属-氧键.

\section{2 苯甲醇电催化氧化性能测试}

为进一步验证其构效关系，我们对 $\mathrm{Ni}-\mathrm{MOF}$ 和
$\mathrm{Ni}(\mathrm{OH})_{2}$ 纳米片进行了一系列的电化学性能测试以评估 其催化活性. 所有测试均在 $1.0 \mathrm{M} \mathrm{KOH}$ 电解液中采用 三电极系统进行测量. 图 $4 \mathrm{a}$ 为 $\mathrm{Ni}-\mathrm{MOF}$ 和 $\mathrm{Ni}(\mathrm{OH})_{2}$ 纳米 片在不同电解质溶液中的LSV曲线. 在 $1.0 \mathrm{M} \mathrm{KOH}$ 中, $\mathrm{Ni}-\mathrm{MOF}$ 和 $\mathrm{Ni}(\mathrm{OH})_{2}$ 均发生析氧反应，在1.33 1.43 V之 间的氧化峰对应于活性物质 $\mathrm{NiOOH}$ 的生成 ${ }^{[31]}$; 在电压 $<1.50 \mathrm{~V}$ 的范围内，其电流密度较小， OER过程缓慢进 

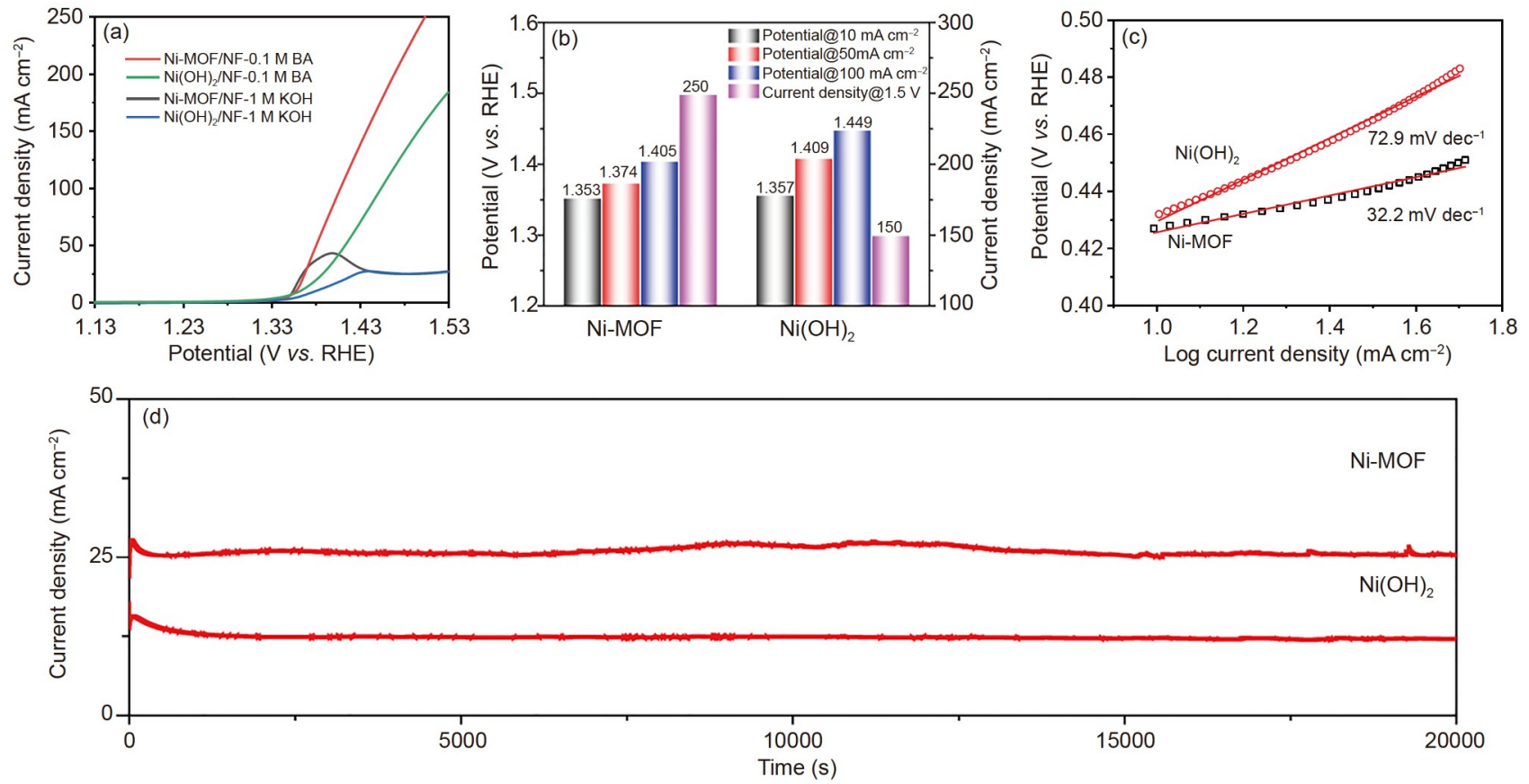

图 4 超薄2D Ni-MOF和 $\mathrm{Ni}(\mathrm{OH})_{2}$ 纳米结构在不同电解质溶液中的(a) LSV曲线, (b) 电流密度与电势变化的柱状图, (c) Tafel曲 线图, (d) $i-t$ 稳定性测试 (网络版彩图)

Figure 4 The (a) LSV curves, (b) histogram of current density-potential, (c) Tafel curves, and (d) $i-t$ measurement of the ultrathin $2 \mathrm{D}$ Ni-MOF and $\mathrm{Ni}(\mathrm{OH})_{2}$ in different electrolyte solutions (color online).

行. 当电解质溶液中添加 $0.1 \mathrm{M}$ 苯甲醇之后, Ni-MOF和 $\mathrm{Ni}(\mathrm{OH})_{2}$ 表现出明显的电流增强 $\left(\mathrm{Ni}-\mathrm{MOF}\right.$ 和 $\mathrm{Ni}(\mathrm{OH})_{2}$ 的 $\mathrm{EBO}$ 效率分别为 $85.0 \%$ 和 $82.4 \%, \mathrm{EBO}$ 效率是用于 $\mathrm{EBO}$ 反应的电荷量占总电荷量的百分比 $E=\left(Q_{(\mathrm{EBO})}-Q_{(\mathrm{OER})}\right) /$ $\left.Q_{(\mathrm{EBO})}\right)^{[32]}$, 表明苯甲醇分子被氧化, 在电催化EBO反应 过程中， $\mathrm{Ni}-\mathrm{MOF}$ 和 $\mathrm{Ni}(\mathrm{OH})_{2}$ 的起峰电位与OER过程中 活性物质 $\mathrm{NiOOH}$ 生成的电位几乎一致，与文献报道结 果一致 ${ }^{[9,33]}$, 充分证明氧化过程产生的 $\mathrm{NiOOH}$ 也是苯 甲醇氧化过程的活性物质. $\mathrm{EBO}$ 的具体参数如图 $4 \mathrm{~b}$ 所 示, 与 $\mathrm{Ni}(\mathrm{OH})_{2}$ 相比, $\mathrm{Ni}-\mathrm{MOF}$ 具有良好的催化活性, 可 以有效降低电催化反应的过电势，在电流密度为 $50 \mathrm{~mA} \mathrm{~cm}{ }^{-2}$ 时, Ni-MOF所需要的氧化电势比 $\mathrm{Ni}(\mathrm{OH})_{2}$ 低 $35 \mathrm{mV}$; 在 $1.50 \mathrm{~V}$ 时, Ni-MOF电极可以实现高效的 $\mathrm{EBO}$ 反应, 电流密度高达 $250 \mathrm{~mA} \mathrm{~cm}{ }^{-2}$, 比 $\mathrm{Ni}(\mathrm{OH})_{2}$ 电极 高 $100 \mathrm{~mA} \mathrm{~cm}{ }^{-2}$, 这一结果优于大多数电催化剂, 且与 已报道的高性能催化剂性能接近(表1 $)^{[8,13,14,34-38]}$. 图4c 展示的是不同样品的 Tafel斜率, $\mathrm{Ni}-\mathrm{MOF}$ 和 $\mathrm{Ni}(\mathrm{OH})_{2}$ 电 极的Tafel斜率分别为 32.2 和 $72.9 \mathrm{mV} \mathrm{dec}{ }^{-1}, \mathrm{Ni}-\mathrm{MOF}$ 电 极表现出低于 $\mathrm{Ni}(\mathrm{OH})_{2}$ 电极的 Tafel斜率, 表明其对电催 化本甲醇氧化具有更快的反应动力学 ${ }^{[26,27]}$. 此外, 稳定
性是实际应用的另一关键参数 ${ }^{[39-42]}$. 对两种催化剂样 品在 $1.42 \mathrm{~V}$ 电位下进行了 $i-t$ 测量, 结果如图 $4 \mathrm{~d}$ 所示, $\mathrm{Ni}-\mathrm{MOF}$ 表现出良好的稳定性，经过 $20000 \mathrm{~s}$ 的持续电 解测量, $\mathrm{Ni}-\mathrm{MOF}$ 的电流密度仍然可以达到 $25 \mathrm{~mA} \mathrm{~cm}^{-2}$, 接近 $\mathrm{Ni}(\mathrm{OH})_{2}$ 电极的 2 倍, 且经过 $20000 \mathrm{~s}$ 的电解几乎无衰减.

样品的电催化活性主要取决于电催化剂的固有活 性位点和活性位点的数量 ${ }^{[3,44]} . \mathrm{Ni}-\mathrm{MOF}$ 和 $\mathrm{Ni}(\mathrm{OH})_{2}$ 纳 米片均具有超薄二维结构, 活性位点得到了有效的暴 露. 为进一步定量的刻画活性位点的数量影响，通过 在非法拉第过程的电位范围内采集不同样品的循环伏 安图进行双电层电容评估, $C_{\mathrm{dl}}$ 与电化学活性表面积成 正比，一定程度上可以反应样品的活性面积和活性位 点. 如图 $5 \mathrm{a} \sim \mathrm{c}$ 所示，计算所得 $\mathrm{Ni}-\mathrm{MOF}$ 和 $\mathrm{Ni}(\mathrm{OH})_{2}$ 纳米 片的 $C_{\mathrm{d} 1}$ 值分别为 9.18 和 $7.41 \mathrm{mF} \mathrm{cm}$. 双电层电容值 十分接近, 表明活性面积相当, 因此不同样品所表现出 的催化活性差异主要来自于本征活性的不同 ${ }^{[21]}$. 在 $\mathrm{Ni}-\mathrm{MOF}$ 电极中 $\mathrm{Ni}^{2+}$ 具有更高的结合能, 趋向于高价氧 化态, 更易于在电催化过程中向活性物质的转化, 有利 于加速反应动力学, 提升电催化活性和效率. 
表 1 不同体系苯甲醇催化氧化反应对照

Table 1 The comparison of the catalytic oxidation of the benzyl alcohol

\begin{tabular}{|c|c|c|c|c|}
\hline 催化反应 & 催化剂 & $\begin{array}{c}\text { 最大电流密度 }\left(\mathrm{mA} \mathrm{cm}^{-2}\right) @ \text { 对应电压 }(\mathrm{V} \\
v s . \mathrm{RHE})\end{array}$ & 反应产物 & 参考文献 \\
\hline 电催化 & $\mathrm{Ni}-\mathrm{MOF}$ & 250@1.50 V & 苯甲酸 & 本文 \\
\hline 电催化 & $\mathrm{Ni}(\mathrm{OH})_{2}$ & 180@1.53 V & 苯甲酸 & 本文 \\
\hline 电催化 & $\mathrm{H}_{5} \mathrm{PMo}_{10} \mathrm{~V}_{2} \mathrm{O}_{40} @ \mathrm{CTF}$ & $12 @ 12 \mathrm{~V}\left(v s . \mathrm{Ag} / \mathrm{Ag}^{+}\right)$ & 苯甲醛 & {$[34]$} \\
\hline 电催化 & $\mathrm{Co}-\mathrm{Ni}-\mathrm{OH} / \mathrm{NF}$ & 400@ 1.45 V & 苯甲酸 & {$[8]$} \\
\hline 电催化 & $\mathrm{Co}_{3} \mathrm{O}_{4} / \mathrm{NF}$ & 86@1.50 V & 苯甲酸 & {$[35]$} \\
\hline 电催化 & 一维 $\mathrm{Co}_{3} \mathrm{O}_{4}$ 纳米结构 & 8@2.5 V (vs. Hg/HgO) & 苯甲酸 & {$[13]$} \\
\hline 电催化 & $\mathrm{Fe} / \mathrm{Co}$ (氧化物) & $100 @ \sim 1.55 \mathrm{~V}$ & 苯甲酸 & {$[14]$} \\
\hline 电催化 & 多孔六方相Ni纳米结构 & $100 @>1.65 \mathrm{~V}$ & 苯甲酸 & [36] \\
\hline 光电催化 & $\mathrm{Bi}_{2} \mathrm{MoO}_{6} @ \mathrm{TiO}_{2}$ & $0.8 @>2$ V (vs. NHE) & 苯甲醛 & {$[37]$} \\
\hline 光电催化 & $\mathrm{TiO}_{2} / \mathrm{Ti}_{3} \mathrm{C}_{2}$ & $<1.2 @>1.98$ V (vs. NHE) & 苯甲醛 & {$[38]$} \\
\hline
\end{tabular}
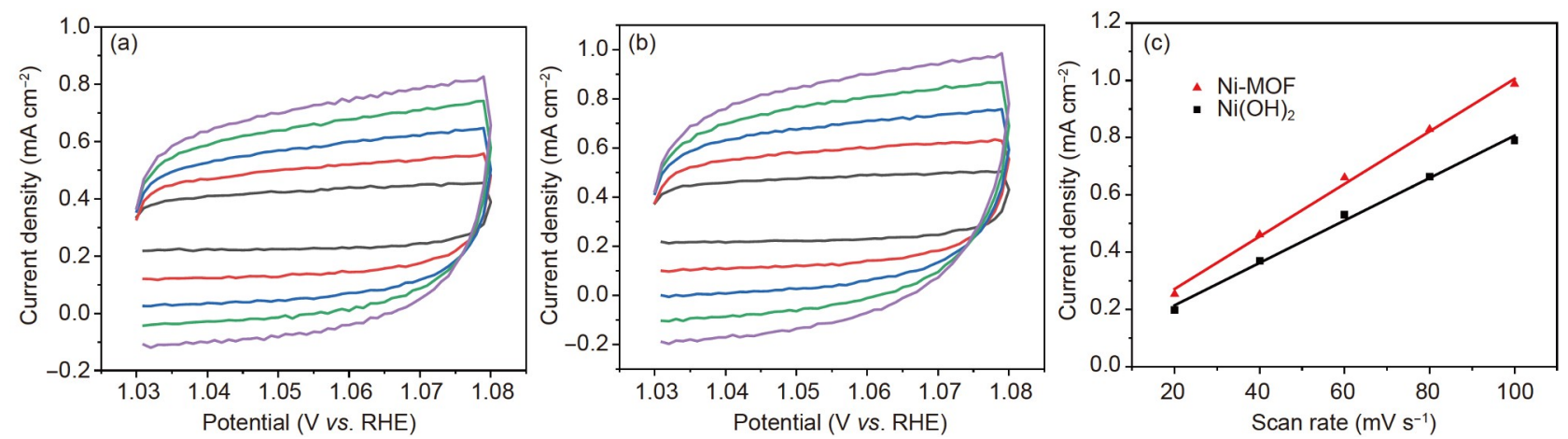

图 5 超薄2D (a) Ni-MOF和(b) $\mathrm{Ni}(\mathrm{OH})_{2}$ 纳米片在 $1.0 \mathrm{M} \mathrm{KOH}$ 中的循环伏安曲线, (c) 不同电催化剂的双电层电容拟合图 (网络 版彩图)

Figure $5 \mathrm{CV}$ curves of the ultrathin 2D (a) Ni-MOF and (b) $\mathrm{Ni}(\mathrm{OH})_{2}$ nanosheets in $1.0 \mathrm{M} \mathrm{KOH}$. (c) The $C_{\mathrm{dl}}$ fitting results of different electrocatalysts (color online).

为表征电催化的产物, 对电解后的样品进行浓盐 酸酸化处理，待析出晶体后分离. 通过XRD和NMR对 电催化所得固体产物进行表征，结果如图6所示. 图6a 和b所给出的XRD谱图是分别在 $\mathrm{Ni}-\mathrm{MOF}$ 和 $\mathrm{Ni}(\mathrm{OH})_{2}$ 电 催化苯甲醇氧化后的产物, 产物均为白色晶体(如插图 所示), 且晶体结构一致, 与苯甲酸的标准卡片(JCPDS No. 12-0853)相匹配, $8^{\circ}$ 和 $16^{\circ}$ 左右的强峰分别对应于 苯甲酸晶体的(200)和(400)晶面, 充分说明电催化苯甲 醇氧化的产物为苯甲酸. 同时, 通过 ${ }^{1} \mathrm{H}$ NMR谱对所得 产物的组成进行进一步研究, 如图6c和 d所示, 从7到 $8.5 \mathrm{ppm}$ 之间的四个强峰可归于苯环上取代基对位、 邻位、间位的三种氢原子的化学位移, $12 \mathrm{ppm}$ 附近的 鼓包峰为-COOH基团中H的化学位移. 因此可以进一
步证明, 两种催化体系所得的固体产物为苯甲酸. 在 $\mathrm{Ni}-\mathrm{NOF}$ 和 $\mathrm{Ni}(\mathrm{OH})_{2}$ 的催化体系中, 苯甲酸的最终产量 分别为 204.6 和 $71.2 \mathrm{mg}$, 充分说明了 $\mathrm{Ni}-\mathrm{NOF}$ 是一种具 有优异电催化苯甲醇氧化的电催化剂材料.

\section{4 结论}

本工作报道了一种温和的溶剂热法制备具有高价 氧化态的超薄二维Ni-MOF纳米片阵列及其在电催化 苯甲醇氧化方面的应用. 作为一种高性能的EBO催化 剂, 2D Ni-MOF展现出较低的过电位和优异的稳定 性, 仅需要约 $1.50 \mathrm{~V}$ 的电位即可达到 $250 \mathrm{~mA} \mathrm{~cm}^{-2}$, $\mathrm{EBO}$ 效率达 $85 \%$; 在 $1.42 \mathrm{~V}$ 电压下, 连续电解 $20000 \mathrm{~s}$ 

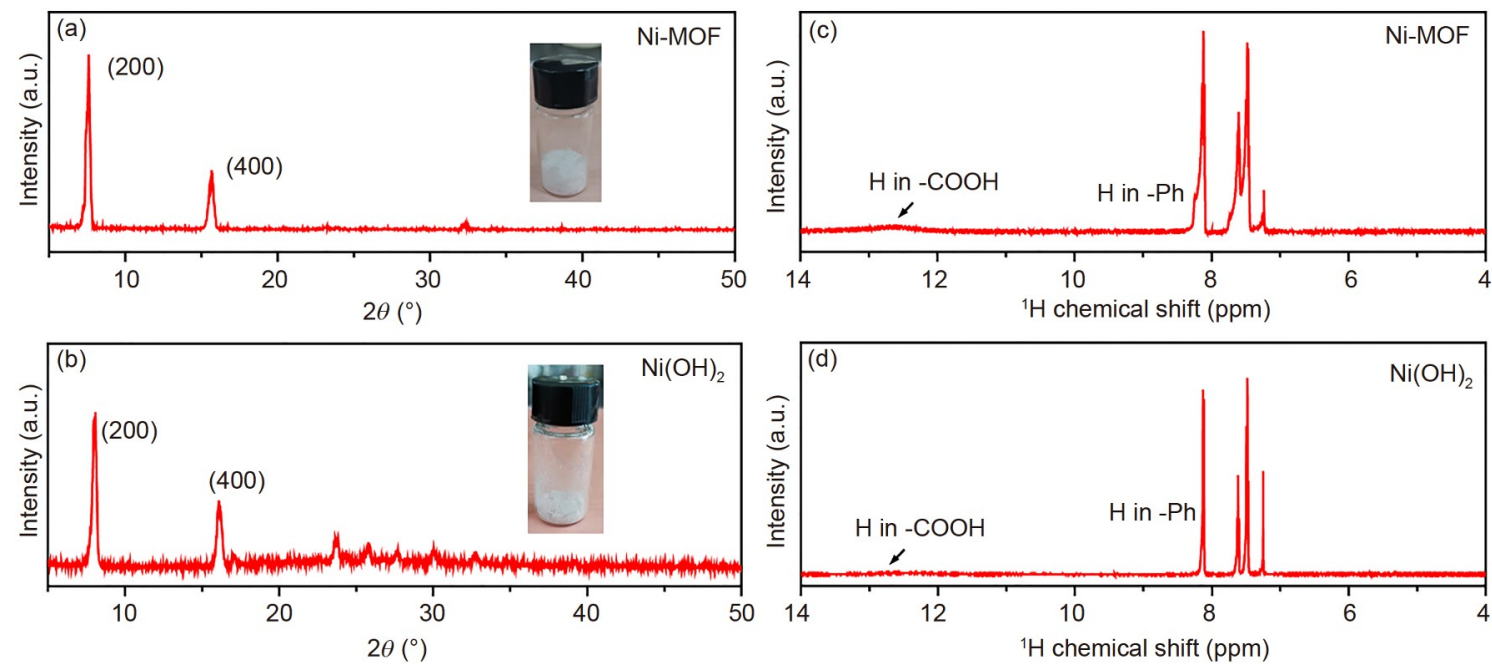

图 6 电催化产物的XRD图谱 $(a, b)$ 和NMR谱图 (c, d), 插图为电催化所得产物苯甲酸的照片 (网络版彩图)

Figure 6 The XRD patterns (a, b) and NMR spectra (c, d) of the product obtained from the different electrocatalysts. The insets of (a, b) were the digital photos of the as-prepared $\mathrm{Ph}-\mathrm{COOH}$ (color online).

后, 可保留 $25 \mathrm{~mA} \mathrm{~cm}^{-2}$ 的电流密度, 接近超薄 $\mathrm{Ni}(\mathrm{OH})_{2}$ 纳米片的两倍. 机理研究表明, Ni-MOF纳米 片中Ni具有更高的氧化态，在EBO反应中更容易转化 成活性位点 $\mathrm{NiOOH}$, 使得其在较低的电位下, 便能有
效催化苯甲醇的电氧化. 这一结果为进一步设计高 性能电催化苯甲醇氧化反应的电催化剂提供了全新 的视角, 为电解混合水同时生产精细化学品提供了 参考.

\section{参考文献}

1 Duan J, Chen S, Zhao C. Nat Commun, 2017, 8: 15341

2 You B, Liu X, Jiang N, Sun Y. J Am Chem Soc, 2016, 138: 13639-13646

3 Song L, Fu N, Ernst BG, Lee WH, Frederick MO, DiStasio Jr. RA, Lin S. Nat Chem, 2020, 12: 747-754

4 Verma S, Lu S, Kenis PJA. Nat Energy, 2019, 4: 466-474

5 Zhang X, Tian F, Gao M, Yang W, Yu Y. Chem Eng J, 2022, 428: 132628

6 Geng S, Tian F, Li M, Liu Y, Sheng J, Yang W, Yu Y, Hou Y. Nano Res, 2021, 15 doi:10.1007/s12274-021-3755-7

7 Zhao S, Wang Y, Dong J, He CT, Yin H, An P, Zhao K, Zhang X, Gao C, Zhang L, Lv J, Wang J, Zhang J, Khattak AM, Khan NA, Wei Z, Zhang J, Liu S, Zhao H, Tang Z. Nat Energy, 2016, 1: 16184

8 Huang H, Yu C, Han X, Huang H, Wei Q, Guo W, Wang Z, Qiu J. Energy Environ Sci, 2020, 13: 4990-4999

9 Zhu X, Dou X, Dai J, An X, Guo Y, Zhang L, Tao S, Zhao J, Chu W, Zeng XC, Wu C, Xie Y. Angew Chem Int Ed, 2016, 55: 12465-12469

10 Deng J, Chen S, Yao N, Wang Q, Li J, Wei Z. Appl Catal B-Environ, 2020, 277: 119175

11 Liu Z, Zhang C, Liu H, Feng L. Appl Catal B-Environ, 2020, 276: 119165

12 You B, Sun Y. Acc Chem Res, 2018, 51: 1571-1580

13 Yin Z, Zheng Y, Wang H, Li J, Zhu Q, Wang Y, Ma N, Hu G, He B, Knop-Gericke A, Schlögl R, Ma D. ACS Nano, 2017, 11: 12365-12377

14 Huang Y, Yang R, Anandhababu G, Xie J, Lv J, Zhao X, Wang X, Wu M, Li Q, Wang Y. ACS Energy Lett, 2018, 3: 1854-1860

15 Yi FY, Zhang R, Wang H, Chen LF, Han L, Jiang HL, Xu Q. Small Methods, 2017, 1: 1700187

16 Zhao R, Liang Z, Zou R, Xu Q. Joule, 2018, 2: 2235-2259

17 Rodenas T, Luz I, Prieto G, Seoane B, Miro H, Corma A, Kapteijn F, Llabrés I Xamena FX, Gascon J. Nat Mater, 2015, 14: 48-55

18 Yuan M, Wang R, Fu W, Lin L, Sun Z, Long X, Zhang S, Nan C, Sun G, Li H, Ma S. ACS Appl Mater Interfaces, 2019, 11: 11403-11413

19 Zhao M, Lu Q, Ma Q, Zhang H. Small Methods, 2017, 1: 1600030 
Peng Y, Li Y, Ban Y, Jin H, Jiao W, Liu X, Yang W. Science, 2014, 346: 1356-1359

Zhu D, Guo C, Liu J, Wang L, Du Y, Qiao SZ. Chem Commun, 2017, 53: 10906-10909

Hu X, Zhu Z, Cheng F, Tao Z, Chen J. Nanoscale, 2015, 7: 11833-11840

Xia H, Zhang J, Yang Z, Guo S, Guo S, Xu Q. Nano-Micro Lett, 2017, 9: 43

Park J, Hinckley AC, Huang Z, Feng D, Yakovenko AA, Lee M, Chen S, Zou X, Bao Z. J Am Chem Soc, 2018, 140: 14533-14537

Sun Z, Lin L, Yuan M, Li H, Sun G, Nan C, Ma S, Yang X. Electrochim Acta, 2018, 281: 420-428

Yan W, Guo Z, Xu H, Lou Y, Chen J, Li Q. Mater Chem Front, 2017, 1: 1324-1330

Bao S, Wang Y, Wei Z, Yang W, Yu Y. J Hazard Mater, 2022, 424: 127370

Zhang X, Tian F, Lan X, Liu Y, Yang W, Zhang J, Yu Y. Chem Eng J, 2022, 429: 132588

Zhang X, Yang W, Gao M, Liu H, Li K, Yu Y. Green Energy Environ, 2022, 7: 66-74

Zhang X, Dong P, Lee JI, Gray JT, Cha YH, Ha S, Song MK. Energy Storage Mater, 2019, 17: 167-177

Sun Z, Wang X, Yuan M, Yang H, Su Y, Shi K, Nan C, Li H, Sun G, Zhu J, Yang X, Chen S. ACS Appl Mater Interfaces, 2020, 12: 23896-23903

Ji RY, Chan DS, Jow JJ, Wu MS. Electrochem Commun, 2013, 29: 21-24

Yuan M, Wang R, Sun Z, Lin L, Yang H, Li H, Nan C, Sun G, Ma S. Inorg Chem, 2019, 58: 11449-11457

Li Z, Zhang J, Jing X, Dong J, Liu H, Lv H, Chi Y, Hu C. J Mater Chem A, 2021, 9: 6152-6159

Cao Y, Zhang D, Kong X, Zhang F, Lei X. J Mater Sci, 2021, 56: 6689-6703

You B, Liu X, Liu X, Sun Y. ACS Catal, 2017, 7: 4564-4570

Bao X, Li H, Wang Z, Tong F, Liu M, Zheng Z, Wang P, Cheng H, Liu Y, Dai Y, Fan Y, Li Z, Huang B. Appl Catal B-Environ, 2021, 286: 119885

Zhou Z, Xie YN, Zhu W, Zhao H, Yang N, Zhao G. Appl Catal B-Environ, 2021, 286: 119868

Mao B, Guo D, Qin J, Meng T, Wang X, Cao M. Angew Chem Int Ed, 2018, 57: 446-450

Mao B, Sun P, Jiang Y, Meng T, Guo D, Qin J, Cao M. Angew Chem Int Ed, 2020, 59: 15232-15237

Yang H, Yuan M, Sun Z, Wang D, Lin L, Li H, Sun G. ACS Sustain Chem Eng, 2020, 8: 8348-8355

Jiang B, Cheong WC, Tu R, Sun K, Liu S, Wu K, Shang H, Huang A, Wang M, Zheng L, Wei X, Chen C. Sci China Mater, 2021, 64: 2729-2738

Yang H, Yuan M, Wang D, Sun Z, Li H, Sun G. ACS Appl Energy Mater, 2021, 4: 8563-8571

Yang Y, Yuan M, Li H, Sun G, Ma S. Electrochim Acta, 2018, 281: 198-207 


\title{
Ultrathin two-dimensional Ni-MOF with high-valence oxidation states as high-performance electrocatalytic catalysts for benzyl-alcohol oxidation
}

\author{
Mengwei Yuan ${ }^{1,3}$, Yayin $\mathrm{Li}^{1}$, Zemin $\operatorname{Sun}^{1,2}$, Genban $\operatorname{Sun}^{1,2^{*}}$ \\ ${ }^{1}$ Beijing Key Laboratory of Energy Conversion and Storage Materials, College of Chemistry, Beijing Normal University, Beijing 100875, China \\ ${ }^{2}$ Center for Advanced Materials Research, Advanced Institute of Natural Sciences, Beijing Normal University, Zhuhai 519087, China \\ ${ }^{3}$ Department of Physics and Applied Optics Beijing Area Major Laboratory, Beijing Normal University, Beijing 100875, China
}

\begin{abstract}
Ultrathin two-dimensional metal-organic frameworks (2D MOFs) have large specific surface area and open catalytically-active centers, which have potential applications in electrocatalytic reactions. This work reports the ultrathin 2D Ni-MOF nanosheet arrays with open sites prepared by a mild solvothermal method and their applications in electrocatalytic benzyl-alcohol oxidation (EBO). As a high-performance EBO electrocatalyst, the 2D Ni-MOF shows low overpotential and superior durability. It only needs a potential of about $1.50 \mathrm{~V}$ ( $v s$. reversible hydrogen electrode (RHE)) to reach $250 \mathrm{~mA} \mathrm{~cm}^{-2}$, and the oxidation efficiency is more than $85 \%$. Compared with the $\left.2 \mathrm{D} \mathrm{Ni(OH}\right)_{2}$ nanosheet arrays with the same ultrathin structure, the Ni-MOF exhibits good activity; its reaction overpotential is reduced by nearly $35 \mathrm{mV}\left(@ 50 \mathrm{~mA} \mathrm{~cm}^{-2}\right)$ and it has faster reaction kinetics. When benzyl alcohol oxidation is carried out at the same constant potential, the Ni-MOF has a higher response current. After continuous electrolysis for 20,000 s, the current density of $25 \mathrm{~mA} \mathrm{~cm}{ }^{-2}$ can be retained, which is nearly twice as much as that of the $\mathrm{Ni}(\mathrm{OH})_{2}$ nanosheets. It is shown that $\mathrm{Ni}$ in the Ni-MOF nanosheets has a higher oxidation state and is easier to be converted to $\mathrm{NiOOH}$ in EBO reactions, so that it can effectively catalyze the electrooxidation reaction of benzyl alcohol at a lower potential. This result will provide a new perspective for the further design of electrocatalysts with high performances for EBO reactions.
\end{abstract}

Keywords: ultrathin two-dimensional structure, two-dimensional metal-organic frameworks, benzyl alcohol oxidation, electrocatalysts, high-valence oxidation state.

doi: $10.1360 /$ SSC-2021-0262 